\title{
The efficacy of homemade tolcapone in the treatment of patients with Parkinson's disease
}

\author{
PEI-LAN ZHANG, YU-XIN WANG, YAN CHEN, CHEN-HAO ZHANG and CHEN-HUA LI \\ Department of Neurology, Tianjin Huanhu Hospital, Tianjin 300350, P.R. China
}

Received June 21, 2017; Accepted October 16, 2017

DOI: $10.3892 /$ etm.2017.5377

\begin{abstract}
The aim of the study was to investigate the efficacy of homemade tolcapone in treatment of patients with Parkinson's disease (PD). Eighty patients with PD were subjected to randomized, double-blind, placebo-controlled and parallel-group study. PD patients were randomly divided into the tolcapone treatment group (41 cases) and placebo group (39 cases). Each patient received 1 pill of tolcapone or placebo, 3 times per day for 26 weeks. Efficacy was evaluated using the internationally used unified Parkinson's disease rating scale (UPDRS) scoring system for PD symptoms. After the treatment for 26 weeks, the cognitive function, tremor, muscle stiffness, voluntary movement and autonomic nerve symptoms were compared between the two groups using UPDRS scores. Compared with the placebo group, cognitive function, muscle stiffness and voluntary movement reduction were significantly improved in patients of the tolcapone group $(\mathrm{P}<0.05)$. However, no significant differences in UPDRS scores of autonomic nerve symptoms and tremor were found between two groups after treatment $(\mathrm{P}>0.05)$. Tolcapone, a catechol- $O$-methyl transferase (COMT) inhibitor, can improve the motor function of patients with PD, especially exercise and muscle stiffness. Tolcapone can also improve the cognitive function of patients.
\end{abstract}

\section{Introduction}

Parkinson's disease (PD) is a disease caused by the degeneration of nigral dopamine neurons in the midbrain and the reduction of striatum dopamine, with static tremor, voluntary movement reduction, muscle stiffness, postural reflex reduction and autonomic nerve dysfunction constituting the main clinical manifestations. L-Dopa and its compound preparation is the most commonly used drugs that can effectively relieve those symptoms, but the efficacy is gradually reduced after

Correspondence to: Dr Pei-Lan Zhang, Department of Neurology, Tianjin Huanhu Hospital, 6 Jizhao Road, Jinnan, Tianjin 300350, P.R. China

E-mail: peilanzhang601@163.com

Key words: tocapine, tolcapone, catechol-o-methyl transferase inhibitor, Parkinson's disease long-term application $(1,2)$. Following treatment of L-Dopa compound preparation for 5-7 years, most PD patients show reactive movement fluctuations and dysmotility. Therefore, how to maintain the concentration of plasma L-Dopa, extend its half-life and develop drugs with a higher concentration of striatum dopamine has become a hot research field in recent years $(3,4)$.

Catechol- $O$-methyl transferase (COMT) is an enzyme that is directly associated with the metabolism of L-Dopa. Novel selective COMT inhibitors tolcapone and entacapone have shown great potential as adjuvant therapy for L-Dopa (5). These drugs can extend the half-life of L-Dopa and stabilize the concentration of L-Dopa in plasma to allow more L-Dopa to enter the brain tissue, thereby elongating the effective acting time. Therefore, these drugs have become a new target for the treatment of PD. Tolcapone is one of the first batch of drugs approved by the FDA to treat PD (2), and it is also the only drug that can inhibit COMT in brain and peripheral tissue. Tolcapone was once named the best novel drug by the International Society for Pharmaceutical Engineering in 1997 (6).

The aim of the study was to investigate the efficacy of homemade tolcapone in treatment of patients with PD. The results showed that, tolcapone, a COMT inhibitor, can improve the motor function of patients with $\mathrm{PD}$, especially exercise and muscle stiffness. Tolcapone can also improve the cognitive function of patients.

\section{Materials and methods}

General information. Eighty patients with PD were randomly divided into the tolcapone group (experimental) and placebo group (control group). There were 41 patients in the experimental group including 21 males and 20 females (average age of $63.47 \pm 9.58$ years). There were 39 patients in experimental group including 26 males and 13 females with an average age of $64.13 \pm 8.71$ years. No significant differences in age, gender, disease history, disease duration and UPDRS score of initial symptoms were found between the two groups $(\mathrm{P}>0.05)$.

Inclusion criteria. The inclusion criteria for the study were: i) Patients were diagnosed with idiopathic PD, grade 1-4 in improved Hoehn and Yahr grading, with no other known or suspected cause of PD; ii) age ranged from 40 to 70 years; iii) patients should show satisfactory response to L-Dopa when L-Dopa treatment was applied, and the stable dose of L-Dopa 
Table I. Comparison of cognitive function UPDRS score at different stages (mean \pm SD).

\begin{tabular}{|c|c|c|c|c|c|c|c|}
\hline Groups & Basic value & Week 4 & Week 8 & Week 12 & Week 16 & Week 21 & Week 26 \\
\hline Experimental group & $1.49 \pm 0.90$ & $1.48 \pm 1.19$ & $1.44 \pm 1.23$ & $1.56 \pm 1.18$ & $1.63 \pm 1.20$ & $1.46 \pm 1.16$ & $1.44 \pm 1.16$ \\
\hline Control group & $1.79 \pm 1.06$ & $1.85 \pm 1.14$ & $1.64 \pm 1.09$ & $1.59 \pm 1.07$ & $1.41 \pm 0.99$ & $1.49 \pm 0.97$ & $1.74 \pm 0.99$ \\
\hline
\end{tabular}

${ }^{*} \mathrm{P}<0.05$, compared with control group.

Table II. Comparison of autonomic nerve UPDRS scores at different stages mean \pm SD.

\begin{tabular}{lcccccccc} 
Groups & Basic value & Week 4 & Week 8 & Week 12 & Week 16 & Week 21 & Week 26 & $\begin{array}{c}\text { basic value and } \\
\text { value of week 26 }\end{array}$ \\
\hline Experimental group & $1.83 \pm 1.60$ & $1.95 \pm 1.66$ & $2.02 \pm 1.62$ & $1.93 \pm 1.79$ & $1.80 \pm 1.81$ & $2.02 \pm 1.78$ & $1.90 \pm 1.85$ & $0.07 \pm 1.29$ \\
Control group & $2.03 \pm 1.22$ & $1.90 \pm 1.39$ & $2.08 \pm 1.33$ & $2.28 \pm 1.41$ & $2.13 \pm 1.38$ & $2.28 \pm 1.39$ & $2.13 \pm 1.26$ & $0.10 \pm 0.95$
\end{tabular}

Table III. Comparison of tremor UPDRS scores at different stages mean \pm SD.

\begin{tabular}{|c|c|c|c|c|c|c|c|c|}
\hline Groups & Basic value & Week 4 & Week 8 & Week 12 & Week 16 & Week 21 & Week 26 & $\begin{array}{c}\text { Difference between } \\
\text { basic value and } \\
\text { value of week } 26\end{array}$ \\
\hline Experimental group & $5.02 \pm 3.76$ & $4.14 \pm 3.23$ & $3.85 \pm 3.49$ & $3.22 \pm 2.92$ & $3.41 \pm 3.03$ & $3.22 \pm 2.74$ & $3.29 \pm 2.99$ & $1.73 \pm 3.03$ \\
\hline Control group & $5.23 \pm 3.59$ & $4.26 \pm 3.66$ & $3.95 \pm 3.49$ & $4.18 \pm 3.68$ & $4.03 \pm 3.62$ & $4.05 \pm 3.52$ & $3.95 \pm 3.46$ & $1.28 \pm 2.35$ \\
\hline
\end{tabular}

should be maintained for at least 1 month prior to the experiment; iv) signed informed consent; v) willing to cooperate with the examiner; vi) patients with other drug treatments were also included.

Exclusion criteria. The exclusion criteria for the study were: i) Patients with degree II or III atrioventricular block or sick sinus syndrome, heart rate at the state of rest $<50$ times $/ \mathrm{min}$, patients with congestive heart failure, and patients experiencing myocardial infarction or significant coronary heart disease within 6 months; ii) patients with liver and kidney dysfunction. iii) patients with atypical Parkinson's syndrome caused by drugs, metabolic diseases, encephalitis or degenerative diseases (such as progressive supranuclear palsy and multiple system atrophy); iv) patients with a history of allergies to drugs and food; v) with active neoplastic disease; vi) with a history of mental illness or treated with antipsychotic drugs within 6 months; vii) hepatitis B-positive; viii) pregnant women or lactating postpartum women.

Methods. i) The drug and placebo were administered as follow. Tolcapone was taken orally at a dose of $100 \mathrm{mg}$ per time, three times per day. Placebo was also taken orally with 1 pill per time, three times per time; ii) forbidden drugs: antipsychotic and antiepileptic drugs; iii) combined medication: L-Dopa, artane, amantadine and other PD drugs.

Criteria for the determination of efficacy. Criteria for the determination of efficacy were based on UPDRS scoring and improved Hoehn and Yahr grading. The criteria were: i) main efficacy indicators: UPDRS score, changes in UPDRS scores from the 2 nd visit to the 7 th visit were analyzed. ii) Time-points for the evaluation of efficacy: Efficacy was evaluated before and $4,8,12,16,21$ and 26 weeks after treatment.

Statistical analysis. Data were analyzed using SAS 10.0 software (Chicago, IL, USA). Measurement data were expressed as mean $\pm \mathrm{SD}$, and processed using a Student's t-test. Count data were processed using an $\chi^{2}$ test. UPDRS scores were subjected to the rank-sum test using SPSS 10.0 software. $\mathrm{P}<0.05$ was considered statistically significant.

\section{Results}

Variables. The efficacies of the tolcapone and placebo groups were compared with regard to factors including cognitive function, tremor, muscle stiffness, voluntary movement and autonomic nerve using UPDRS scoring.

Cognitive function score. The cognitive function of the tolcapone group using the UPDRS score was significantly improved at 6 months after treatment compared with the placebo group $(\mathrm{P}<0.05)$ (Table I).

Autonomic nerve score. No significant difference in the autonomic nerve UPDRS score was found between the two groups, indicating that this drug cannot be used to improve autonomic nerve disorders of PD (Table II). 
Table IV. The difference between tremor UPDRS score and basic value at different stages (mean \pm SD).

\begin{tabular}{|c|c|c|c|c|c|c|}
\hline Groups & Week 1-4 & Week 1-8 & Week 1-12 & Week 1-16 & Week 1-21 & Week 1-26 \\
\hline Experimental group & $0.88 \pm 3.24$ & $1.17 \pm 2.04$ & $1.80 \pm 2.58$ & $1.61 \pm 2.46$ & $1.80 \pm 2.71$ & $1.73 \pm 3.03$ \\
\hline Control group & $0.97 \pm 2.19$ & $1.28 \pm 2.18$ & $1.05 \pm 2.31$ & $1.21 \pm 2.40$ & $1.18 \pm 2.22$ & $1.28 \pm 2.35$ \\
\hline
\end{tabular}

Table V. Comparison of muscle stiffness UPDRS scores at different stages (mean \pm SD).

\begin{tabular}{|c|c|c|c|c|c|c|c|c|}
\hline Groups & Basic value & Week 4 & Week 8 & Week 12 & Week 16 & Week 21 & Week 26 & $\begin{array}{c}\text { basic value and } \\
\text { value of week } 26\end{array}$ \\
\hline $\begin{array}{l}\text { Experimental } \\
\text { group }\end{array}$ & $24.78 \pm 15.25$ & $22.78 \pm 14.85$ & $22.41 \pm 15.05$ & $21.90 \pm 15.22$ & $21.51 \pm 15.05^{\mathrm{a}}$ & $21.59 \pm 15.42^{\mathrm{a}}$ & $21.7 \pm 15.73^{a}$ & $3.71 \pm 5.34$ \\
\hline Control group & $23.10 \pm 10.86$ & $22.10 \pm 8.71$ & $21.87 \pm 9.20$ & $22.46 \pm 10.60$ & $22.87 \pm 10.68$ & $23.44 \pm 10.66$ & $23.00 \pm 10.52$ & $0.10 \pm 6.97$ \\
\hline
\end{tabular}

${ }^{\mathrm{a}} \mathrm{P}<0.05$, compared with control group.

Table VI. The difference between muscle stiffness UPDRS score and basic value at different stages (mean \pm SD).

\begin{tabular}{lrrrrrr}
\hline Groups & Week 1-4 & Week 1-8 & Week 1-12 & Week 1-16 & Week 1-21 & Week 1-26 \\
\hline Experimental group & $2.00 \pm 5.48$ & $2.377 \pm 5.01$ & $2.88 \pm 5.57$ & $3.27 \pm 5.02$ & $3.20 \pm 5.34$ \\
Control group & $1.00 \pm 6.24$ & $1.23 \pm 6.29$ & $0.64 \pm 6.42$ & $0.23 \pm 6.31$ & $0.33 \pm 7.50$ & $0.10 . \pm 6.97$ \\
\hline
\end{tabular}

$\mathrm{P}<0.05$, compared with control group.

Table VII. Comparison of voluntary movement reduction UPDRS scores at different stages (mean \pm SD).

\begin{tabular}{lcccccccc} 
Groups & Basic value & Week 4 & Week 8 & Week 12 & Week 16 & Week 21 & Week 26 & $\begin{array}{c}\text { basic value and } \\
\text { value of week 26 }\end{array}$ \\
\hline Experimental group & $2.44 \pm 1.53$ & $1.88 \pm 1.42$ & $1.85 \pm 1.93$ & $1.98 \pm 2.08$ & $2.02 \pm 2.15$ & $1.98 \pm 1.99$ & $2.02 \pm 2.04$ & $0.41 \pm 1.66$ \\
Control group & $2.54 \pm 2.38$ & $2.59 \pm 2.10$ & $2.33 \pm 2.22$ & $2.56 \pm 2.04$ & $2.56 \pm 1.92$ & $2.69 \pm 1.84$ & $3.18 \pm 3.03$ & $0.64 \pm 3.04$ \\
\hline
\end{tabular}

$\mathrm{P}<0.01$ or $\mathrm{P}<0.05$, compared with control group.

Table VIII. The difference between voluntary movement reduction UPDRS score and basic value at different stages (mean \pm SD).

\begin{tabular}{|c|c|c|c|c|c|c|}
\hline Groups & Week 1-4 & Week 1-8 & Week 1-12 & Week 1-16 & Week 1-21 & Week 1-26 \\
\hline Experimental group & $0.56 \pm 1.12$ & $0.59 \pm 1.60$ & $0.46 \pm 1.61^{\mathrm{a}}$ & $0.41 \pm 1.61^{\mathrm{a}}$ & $0.46 \pm 1.61^{\mathrm{a}}$ & $0.41 \pm 1.66^{\mathrm{a}}$ \\
\hline Control group & $0.64 \pm 3.04$ & $0.05 \pm 1.59$ & $0.21 \pm 1.45$ & $0.03 \pm 1.69$ & $0.03 \pm 1.80$ & $0.15 \pm 1.89$ \\
\hline
\end{tabular}

${ }^{\mathrm{a}} \mathrm{P}<0.05$, compared with control group.

Tremor score. No significant difference in tremor UPDRS score was found between the groups, indicating that this drug cannot be used to improve tremor of PD (Tables III and IV).

Muscle stiffness score. No significant difference in the muscle stiffness UPDRS score was found between the two groups. The difference between tremor muscle stiffness score and basic value was significantly improved in the tolcapone group compared with the placebo group from week 16 (Tables V and VI).

Voluntary movement reduction score. A comparison of the voluntary movement reduction UPDRS score showed that the 
voluntary movement reduction UPDRS score of the tolcapone group was significantly lower than that of the placebo group at weeks 21 and $26(\mathrm{P}<0.05)$. Analysis of the difference between tremor muscle stiffness score and basic value showed that voluntary movement reduction in the tolcapone group was significantly improved compared with th eplacebo group from week $12(\mathrm{P}<0.01$ or $\mathrm{P}<0.05)$ (Tables VII and VIII).

\section{Discussion}

In this study, the efficacies of the tolcapone and placebo groups were compared from the aspects of cognitive function, tremor, muscle stiffness, voluntary movement reduction and autonomic nerve using UPDRS scoring, and significant differences in the cognitive function were found between the two groups $(\mathrm{P}<0.05)$. The possible mechanism (7) involved was that, COMT can inactive catecholamine neurotransmitters, such as DA and noradrenaline (NA). The inhibitor of COMT can rapidly and persistently inhibit the degradation of endogenous NA and improve the bioavailability of DA and NA. DA and NA are involved in attention control. DA determines the control level and NA can play a regulatory role to keep the connections between attention, sense of discrimination and creation function. NA deficiency can lead to cognitive function impairment, such as obstacles in attention, memory and learning after coeruleus damage (8). It has been reported that the lack of attention in PD patients is due to damage to the upstream monoamine pathway. Therefore, COMT inhibitors can improve the cognitive function of PD patients by improving the bioavailability of DA and NA (9). Significant differences in muscle stiffness and voluntary movement reduction were also found between the two groups $(\mathrm{P}<0.05)$. Voluntary movement reduction is believed to be associated with the increased muscle tension. However, in recent years, some researchers argued that voluntary movement reduction is the result of the chaos of dynamic mode of the central nervous system, the main obstacle does not seem to be the formation of the movement plan, but rather the implementation thereof $(10,11)$. In other words, the movement plan, which is mainly formed by learning, cannot be automatically performed. Obstacles, which may be correlated with the disorders in the connection between basal ganglia and motor cortex, are mainly manifested in the implementation of complex motor programs. Neurotransmitter is mainly related to the lower level of DA and high level of vanillic acid (m, A), the more obvious the lack of DA, the more serious the symptoms $(12,13)$. The results of the present study may be related to the improvement of L-Dopa bioavailability and the enhanced efficacy of L-Dopa (14).

No significant difference in tremor UPDRS score was found between the two groups. The biochemical basis of static tremor is closely correlated with the imbalance of DA-Ach and 5-HT histamine. Although DA deficiency was improved after treatment, the balance of DA-Ach and 5-HT histamine was not completely corrected; thus, the improvement in tremor is not obvious. Autonomic nerve dysfunction can be manifested by 'greasy face' caused by seborrhoeic dermatitis, hyperhidrosis caused by sweat gland secretion function hyperthyroidism, salivation caused by reduced number of swallowing (caused by mouth, pharynx and palate muscle movement disorder), stubborn constipation caused by digestive tract peristalsis disorder, and orthostatic hypotension caused by sympathetic nervous system dysfunction. The damage of dorsal vagal nucleus is the pathological basis of the autonomic symptoms of the disease. No significant difference in autonomic nerve symptoms between the tolcapone and placebo groups, indicating that the improvement of DA deficiency was not associated with the improvement of dorsal vagal nucleus damage $(15,16)$.

COMT inhibitor tolcapone can significantly improve the motor function of patients with PD, especially exercise reduction and voluntary movement reduction. Tolcapone can also improve the cognitive function of the patient.

\section{References}

1. Marsden CD: Parkinson's disease. J Neurol Neurosurg Psychiatry 57: 672-681, 1994.

2. Zulli C, Sica M, De Micco R, Del Prete A, Amato MR, Tessitore A, Ferraro F and Esposito P: Con-tinuous intra jejunal infusion of levodopa-carbidopa intestinal gel by jejunal extension tube placement through percutaneous endoscopic gastrostomy for patients with advanced Parkinson's disease: A preliminary study. Eur Rev Med Pharmacol Sci 20: 2413-2417, 2016.

3. Wu L, Mu N, Yang F, Zang J and Zheng JP: A study of the non-motor symptoms in early Parkinson's disease with olfactory deficits. Eur Rev Med Pharmacol Sci 20: 3857-3862, 2016.

4. Fahn S: Tolcapone: COMT inhibition for the treatment of Parkinson's disease. Neurology 50 (Suppl 5): S1-S2, 1998.

5. Grünig D, Felser A, Bouitbir J and Krähenbühl S: The catechol$O$-methyltransferase inhibitors tolcapone and entacapone uncouple and inhibit the mitochondrial respiratory chain in HepaRG cells. Toxicol In Vitro 42: 337-347, 2017.

6. Jorga KM, Sedek G, Fotteler B, Zürcher G, Nielsen T and Aitken JW: Optimizing levodopa pharmacokinetics with multiple tolcapone doses in the elderly. Clin Pharmacol Ther 62: 300-310,1997.

7. Lisowska A,Stawińska-Witoszyńska B, BajerskaJ,KrzyżanowskaP and Walkowiak J: Green tea influences intestinal assimilation of lipids in humans: A pilot study. Eur Rev Med Pharmacol Sci 19: 209-214, 2015.

8. Erez A, Li J, Geraghty MT, Ben-Shachar S, Cooper ML, Mensing DE, Vonalt KD, Ou Z, Pursley AN, Chinault AC, Patel A, Cheung SW and Sahoo T: Mosaic deletion 11p13 in a child with dopamine beta-hydroxylase deficiency - case report and review of the literature. Am J Med Genet A 152A: 732-736, 2010.

9. Martinez-Martin P and O'Brien CF: Extending levodopa action: COMT inhibition. Neurology 50 (Suppl 6): S27-S48, 1998.

10. Rajput AH, Martin W, Saint-Hilaire MH, Dorflinger E and Pedder S: Tolcapone improves motor function in parkinsonian patients with the 'wearing-off' phenomenon: A double-blind, placebo-controlled, multicenter trial. Neurology 49: 1066-1071, 1997.

11. Song JH, Zhou PY, Cao ZH, Ding ZG, Chen HX and Zhang GB: Rhythmic auditory stimulation with visual stimuli on motor and balance function of patients with Parkinson's disease. Eur Rev Med Pharmacol Sci 19: 2001-2007, 2015.

12. Kurth MC and Adler CH: COMT inhibition: A new treatment strategy for Parkinson's disease. Neurology 50 (Suppl 5): S3-S14, 1998.

13. Ding W, Ding LJ, Li FF, Han Y and Mu L: Neurodegeneration and cognition in Parkinson's disease: A review. Eur Rev Med Pharmacol Sci 19: 2275-2281, 2015.

14. Cummings JL: Depression and Parkinson's disease: A review. Am J Psychiatry 149: 443-454, 1992.

15. Kaakkola S, Gordin A and Männistö PT: General properties and clinical possibilities of new selective inhibitors of catechol O-methyltransferase. Gen Pharmacol 25: 813-824, 1994.

16. Zhuang JJ, Zheng YH, Xu XW and Zhou L: The ultra early diagnosis of Parkinson's disease by the enhanced substantia nigra echo. Eur Rev Med Pharmacol Sci 19: 4621-4626, 2015.

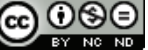

This work is licensed under a Creative Commons Attribution-NonCommercial-NoDerivatives 4.0 International (CC BY-NC-ND 4.0) License. 